\title{
Single Atom Niobium in Graphitic Layers for Enhanced Oxygen Reduction Reaction
}

Junjie Guo ${ }^{1,2}$, Xuefeng Zhang ${ }^{3,4}$, Pengfei Guan ${ }^{5,6}$, Chunjing Liu ${ }^{4}$, Hao Huang ${ }^{4}$, Fanghong Xue ${ }^{4}$, Xinglong Dong ${ }^{4}$, Stephen J. Pennycook ${ }^{1,2}$, Matthew F. Chisholm ${ }^{1}$

${ }^{1}$ Materials Science and Technology Division, Oak Ridge National Laboratory, Oak Ridge, TN 37831, USA.

${ }^{2}$ Department of Materials Science and Engineering, University of Tennessee, Knoxville, TN37996, USA

${ }^{3}$ National Research Council of Canada, 75 Boul. de Mortagne, Boucherville, Québec, J4B 6Y4, Canada.

${ }^{4}$ School of Materials Science and Engineering, Dalian University of Technology, Dalian, Liaoning, 116024, People's Republic of China

${ }^{5}$ Department of Materials Science and Engineering, Johns Hopkins University, Baltimore, MD 21218, USA.

${ }^{6}$ Department of Physics, Tsinghua University, Beijing 100084, People's Republic of China.

Carbides of groups IV through VI ( $\mathrm{Ti}, \mathrm{V}$, and $\mathrm{Cr}$ groups) have long been proposed as substitutes for noble metal-based electrocatalysts in polymer electrolyte fuel cells [1]. Yet their catalytic activity has been extremely limited due to a low density and stability of catalytically active sites. Decreasing the metallic catalyst size is considered an effective way to increase the number of active sites [2]. However, miniaturization to nanometer-scale particles or even to single atoms remains a significant challenge because of aggregation, which can result in drastic loss of catalytic activity.

Here, we report the excellent performance of a niobium-carbon structure for catalyzing the cathodic oxygen reduction reaction (ORR). This unique structure was synthesized using an arc-discharge method. A large number of single niobium atoms and ultra-small clusters were found to be trapped in graphitic layers as identified using state-of-the-art aberration-corrected scanning transmission electron microscopy (STEM, Nion-UltraSTEM100) $[3,4]$. The achievable spatial resolution at a low accelerating voltage of $60 \mathrm{kV}$ is $\sim 1.2 \AA$. The coupled electron energy-loss spectrometer (EELS, Gatan Enfina) has an energy resolution of $0.3 \mathrm{eV}$.

A representative ADF image in Fig. 1(a) reveals a heterogeneous dispersion of sub-nanometer niobium carbide clusters and single niobium atoms (bright region). The graphitic shell with distorted $\{002\}$ planes contains pronounced ripples and mesoporous defects, which were most likely derived from the rapid cooling process. No evidence for the presence of other impurities, such as oxygen, except carbon and niobium was found from EELS, see Fig. 1(b). Furthermore, a $\sim 2 \mathrm{~nm}$ niobium carbide cluster encapsulated in a hollow onion-like carbon shell (Fig. 1(c)) was analysed by EELS elemental mapping, as seen in Figs. 1(d)-(f). This structure not only enhanced the overall conductivity for accelerating the exchange of ions and electrons during the ORR process, but it also suppressed the chemical/thermal coarsening of the active particles.

Experimental results coupled with density functional theory calculations reveal that the single niobium atoms incorporated within the graphitic layers produce a redistribution of $d$-band electrons and become surprisingly active for $\mathrm{O}_{2}$ adsorption and dissociation and also exhibit high stability. 
References:

[1] R.B. Levy and M. Boudart, Science, 81 (1973) 547.

[2] K. Yamamoto, et al. Nat Chem, 1 (2009) 397.

[3] O.L. Krivanek, et al. Nature, 464 (2010) 571.

[4] J. Guo, et al. Small, 8 (2012) 3283.

[5] Acknowledgment: This work was supported by the U.S. Department of Energy (DOE), Basic Energy Sciences (BES), Materials Sciences and Engineering Division, and through a user project supported by ORNL's Shared Research Equipment (ShaRE) User Program, which is also sponsored by DOE-BES.
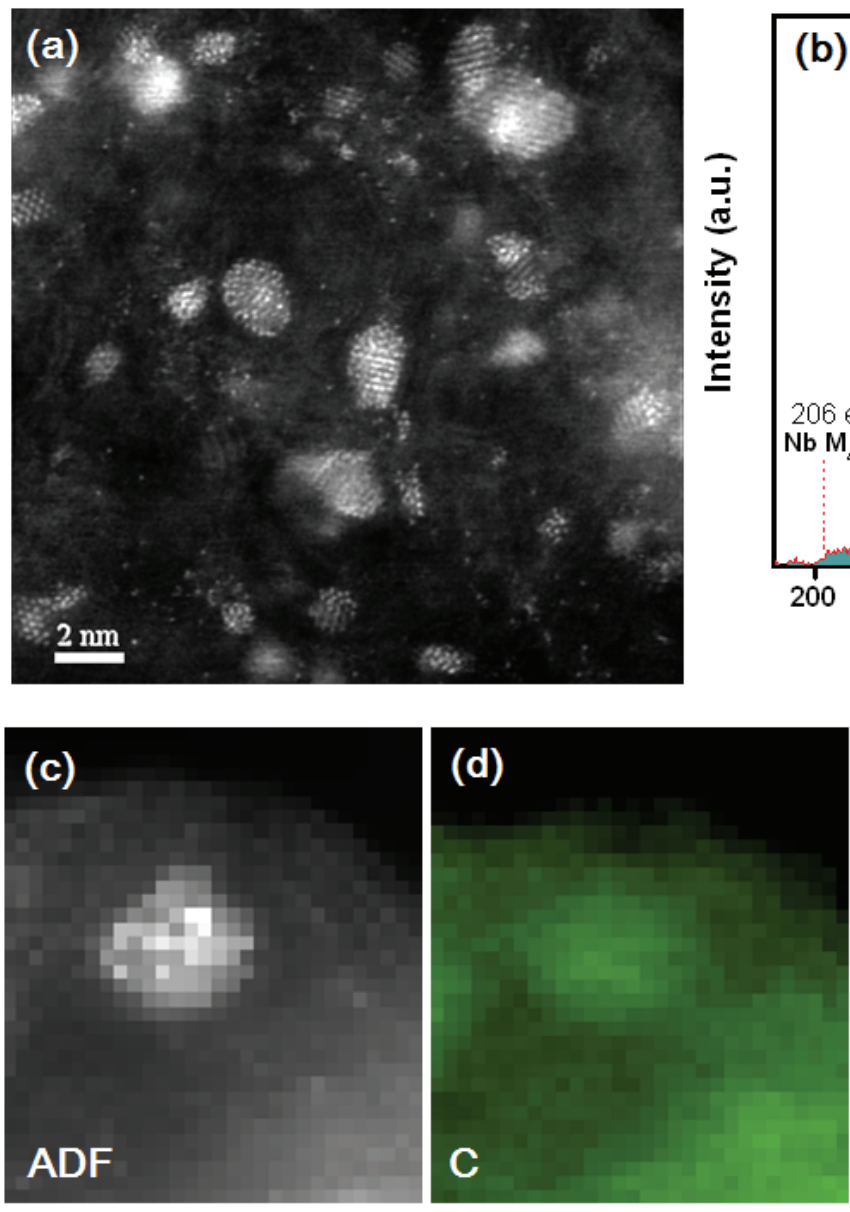
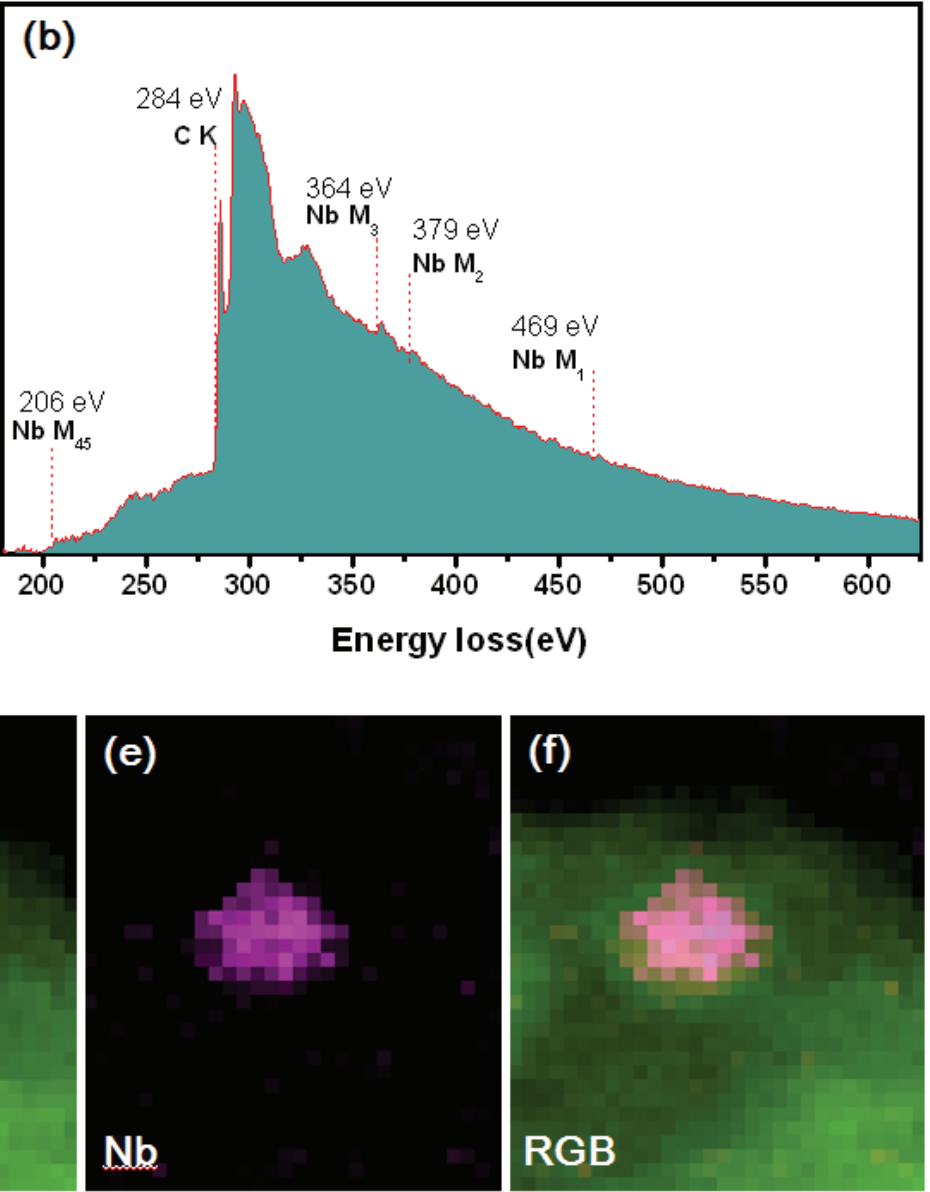

Figure 1. Atomic resolution imaging and EELS mapping of a $\mathrm{Nb}$-in-C complex. (a) ADF image of carbon based catalyst, showing niobium nanoparticles and single atoms (bright regions). (b) EEL spectrum from the particle and surrounding carbon. Simultaneously collected ADF intensity map (c), C composition map (d), $\mathrm{Nb}$ composition map (e), and overlaid $\mathrm{C}$ and $\mathrm{Nb}$ maps (f) from a $\mathrm{Nb}$ carbide particle. 